PROCEEDINGS OF THE

AMERICAN MATHEMATICAL SOCIETY

Volume 132, Number 6, Pages 1639-1647

S 0002-9939(04)07401-5

Article electronically published on January 29, 2004

\title{
EMBEDDINGS OF SOME CLASSICAL BANACH SPACES INTO MODULATION SPACES
}

\author{
KASSO A. OKOUDJOU
}

(Communicated by David R. Larson)

\begin{abstract}
We give sufficient conditions for a tempered distribution to belong to certain modulation spaces by showing embeddings of some Besov-TriebelLizorkin spaces into modulation spaces. As a consequence we have a new proof that the Hölder-Lipschitz space $C^{s}\left(\mathbb{R}^{d}\right)$ embeds into the modulation space $M^{\infty, 1}\left(\mathbb{R}^{d}\right)$ when $s>d$. This embedding plays an important role in interpreting recent modulation space approaches to pseudodifferential operators.
\end{abstract}

\section{INTRODUCTION}

An important question in time-frequency analysis is the choice of a convenient representation together with an adequate measure of the joint time-frequency concentration of a signal or distribution. The Fourier transform provides only nonlocalized frequency information, whereas time-frequency analysis seeks a representation that simultaneously displays the time-domain and frequency-domain behaviors of a signal. The short-time Fourier transform (STFT) or windowed Fourier transform is an important joint time-frequency representation. It shares with the Fourier transform many useful mathematical properties, including linearity and continuity as operators on appropriate spaces. Moreover, properties of the STFT can be transferred to other important time-frequency representations such as the Wigner distribution or the ambiguity function [4]. To quantitatively measure the time-frequency content of a distribution, Feichtinger introduced a class of Banach spaces called the modulation spaces, denoted $\mathcal{M}^{p, q}$ or (for the weighted versions) $\mathcal{M}_{\omega}^{p, q}$, by imposing a mixed $L^{p, q}$ norm on the STFT ([1, [ 8]). The modulation spaces are often the "right spaces" in which to formulate results on time-frequency analysis. We refer to [8] and the references therein for more details about these spaces.

The apparently simple definition of the modulation spaces hides the practical problem of how to decide whether or not a distribution belongs to a given modulation space. In principle one has to estimate the $L^{p, q}$ norm of the STFT, which can be a nontrivial task. Therefore, it is important to understand the relationship between time-frequency content and other properties of distributions, e.g.,

Received by the editors March 22, 2002.

2000 Mathematics Subject Classification. Primary 46E35; Secondary 42 B35.

Key words and phrases. Besov space, modulation space, Sobolev space, short-time Fourier transform, Triebel-Lizorkin space, time-frequency analysis.

The author was partially supported by NSF Grant DMS-9970524. 
smoothness properties. Such relationships may appear in the form of embeddings of certain spaces that measure smoothness and/or decay into modulation spaces. For example, Gröchenig in [7] and Hogan and Lakey in [11] derived sufficient conditions for membership in the modulation space $\mathcal{M}^{1,1}$ (also called the Feichtinger algebra) from certain uncertainty principles related to the STFT. Precisely, it was shown that $L_{a}^{p} \cap \mathcal{F} L_{b}^{q} \subset \mathcal{M}^{1,1}$ under appropriate conditions on $p, q$ and the weight parameters $a, b$, where $L_{a}^{p}$ is a weighted $L^{p}$ space (with weight $(1+|x|)^{a}$ ), and $\mathcal{F} L_{b}^{q}$ is the image of $L_{b}^{q}$ under the Fourier transform. Somewhat more general embeddings involving weighted $L^{p}$ spaces were given by Galperin and Gröchenig in [5]. Another interesting example appears in [10]. There, Heil, Ramanathan and Topiwala proved that $\mathcal{C}^{s}\left(\mathbb{R}^{2 d}\right) \subset \mathcal{M}^{\infty, 1}\left(\mathbb{R}^{2 d}\right)$ for $s>2 d$. This embedding is particularly important in relation to pseudodifferential operator theory. It was shown in 9 that a Weyl or Kohn-Nirenberg pseudodifferential operator $\sigma(D, X)$ is bounded on $\mathcal{M}^{p, p}\left(\mathbb{R}^{d}\right)$, including $\mathcal{M}^{2,2}=L^{2}$ in particular, if the associated symbol $\sigma(\xi, x)$ lies in $\mathcal{M}^{\infty, 1}\left(\mathbb{R}^{2 d}\right)$. Thus this embedding recovers and extends the classical CalderonVaillancourt-type theorem that $\sigma(D, X)$ is bounded on $L^{2}\left(\mathbb{R}^{d}\right)$ if $\sigma(\xi, x) \in \mathcal{C}^{s}\left(\mathbb{R}^{2 d}\right)$ with $s>2 d$; cf. [4, Theorem 2.73].

From these examples, we can see the need for practical and easily checked conditions implying that a distribution belongs to a given modulation space. Our goal in this paper is to provide some sufficient conditions by proving some embeddings of classical Banach spaces such as the Besov, Triebel-Lizorkin, or Sobolev spaces into the modulation spaces. As corollaries, we obtain some embeddings that generalize the embedding from [10] mentioned above, and moreover, we will give an easy sufficient condition for membership of a distribution in $\mathcal{M}^{1,1}$ in the special case of dimension $d=1$.

While writing this paper, H. Feichtinger informed us of the unpublished Ph.D. thesis of Gröbner [6], which also obtains some embeddings of Besov spaces into the modulation spaces. Gröbner's results and techniques are distinct from those presented here. Gröbner constructs a continuous family of Banach spaces having the modulation spaces at one endpoint and the Besov spaces at the other.

Additionally, after our paper was completed we learned that J. Toft 14 has simultaneously obtained some embeddings of Besov spaces into the modulation spaces. Toft's techniques are again different, since he applies Young-type inequalities to derive the embeddings. Additionally, the embeddings obtained are distinct from ours. In particular, Toft does not recover the embedding from [10], as we do.

\section{Definitions AND BACKGROUND}

2.1. The short-time Fourier transform and the modulation spaces. All function spaces will be defined over $\mathbb{R}^{d}$ unless otherwise stated. The Fourier transform of $f \in L^{1}$ is $\hat{f}(\omega)=\int_{\mathbb{R}^{d}} f(t) e^{-2 \pi i t \cdot \omega} d t$. The Fourier transform is an isomorphism of the Schwartz space $\mathbb{S}$ onto itself, and extends to the space $\mathbb{S}^{\prime}$ of tempered distributions by duality. The inverse Fourier transform is $\check{f}(x)=\int_{\mathbb{R}^{d}} f(t) e^{2 \pi i t \cdot x} d t$.

Translation, modulation, and dilation of a function $f$ are

$$
T_{x} f(t)=f(t-x), \quad M_{\omega} f(t)=e^{2 \pi i t \cdot \omega} f(t), \quad \text { and } \quad D_{a} f(t)=|a|^{-d / 2} f(t / a) .
$$


The short-time Fourier transform (STFT) of a function $f$ with respect to a window $g$ is

$$
V_{g} f(x, \omega)=\left\langle f, M_{\omega} T_{x} g\right\rangle=\int_{\mathbb{R}^{d}} e^{-2 \pi i \omega \cdot t} \overline{g(t-x)} f(t) d t,
$$

whenever the integral makes sense. Analogously to the Fourier transform, the STFT extends in a distributional sense to $f, g \in \mathbb{S}^{\prime}$; cf. [4. Prop. 1.42].

Definition 2.1. Given $1 \leq p, q \leq \infty$, and given a window function $g \in \mathbb{S}, \mathcal{M}^{p, q}$ is the space of all distributions $f \in \mathbb{S}^{\prime}$ for which the following norm is finite:

$$
\|f\|_{\mathcal{M}^{p, q}}=\left(\int_{\mathbb{R}^{d}}\left(\int_{\mathbb{R}^{d}}\left|V_{g} f(x, \omega)\right|^{p} d x\right)^{q / p} d \omega\right)^{1 / q}=\left\|V_{g} f\right\|_{L^{p, q}}
$$

with the usual modifications if $p$ or $q$ is infinite.

This definition is independent of the choice of the window $g$ in the sense of equivalent norms. For background and information on the basic properties of the modulation spaces we refer to [1], 2], 3], and [ $]$.

The next proposition, due to Feichtinger [1, on complex interpolation of modulation spaces will be used in the proof of our results.

Proposition 2.2. Let $1 \leq p_{0}<\infty, 1 \leq q_{0}<\infty, 1 \leq p_{1} \leq \infty, 1 \leq q_{1} \leq \infty$, and $\theta \in(0,1)$. If $s=(1-\theta) s_{0}+\theta s_{1}, \frac{1}{p}=\frac{1-\theta}{p_{0}}+\frac{\theta}{p_{1}}$, and $\frac{1}{q}=\frac{1-\theta}{q_{0}}+\frac{\theta}{q_{1}}$, then

$$
\left(\mathcal{M}^{p_{0}, q_{0}}, \mathcal{M}^{p_{1}, q_{1}}\right)_{\theta}=\mathcal{M}^{p, q} \text {. }
$$

2.2. The Besov and Triebel-Lizorkin spaces. Let $\psi \in \mathbb{S}$ be a function such that

Define

$$
\left\{\begin{aligned}
0 \leq \psi(x) \leq 1, & \\
\psi(x)=1, & \text { if } \quad|x| \leq 1 \\
\psi(x)=0, & \text { if } \quad|x| \geq 3 / 2 .
\end{aligned}\right.
$$

$$
\left\{\begin{array}{l}
\phi_{0}(x)=\psi(x) \\
\phi_{1}(x)=\psi\left(\frac{x}{2}\right)-\psi(x) \\
\phi_{k}(x)=\phi_{1}\left(2^{-k+1} x\right), k=2,3, \ldots
\end{array}\right.
$$

Then $\left\{\phi_{k}\right\}_{k=0}^{\infty}$ is a partition of unity, and satisfies $\operatorname{supp}\left(\phi_{k}\right) \subset\left\{x \in \mathbb{R}^{d}: 2^{k-1} \leq\right.$ $\left.|x| \leq 3 \cdot 2^{k-1}\right\}$.

Definition 2.3. Let $s \in \mathbb{R}, 1 \leq q \leq \infty$, and $f \in \mathbb{S}^{\prime}$.

(i) For $1 \leq p<\infty$, the Triebel-Lizorkin space $\mathcal{F}_{p, q}^{s}$ is defined by:

$$
f \in \mathcal{F}_{p, q}^{s} \Longleftrightarrow\|f\|_{\mathcal{F}_{p, q}^{s}}=\left(\int_{\mathbb{R}^{d}}\left(\sum_{k=0}^{\infty} 2^{s k q} \mid\left(\phi_{k} \hat{f}(x)\right)^{q}\right)^{p / q} d x\right)^{1 / p}<\infty .
$$

(ii) For $1 \leq p \leq \infty$, the Besov space $\mathcal{B}_{p, q}^{s}$ is defined by:

$$
f \in \mathcal{B}_{p, q}^{s} \Longleftrightarrow\|f\|_{\mathcal{B}_{p, q}^{s}}=\left(\sum_{k=0}^{\infty} 2^{s k q}\left(\int_{\mathbb{R}^{d}} \mid\left(\phi_{k} \hat{f}(x)\right)^{p} d x\right)^{q / p}\right)^{1 / q}<\infty .
$$

(iii) For $p=\infty$, the Triebel-Lizorkin space $\mathcal{F}_{\infty, q}^{s}$ is defined by:

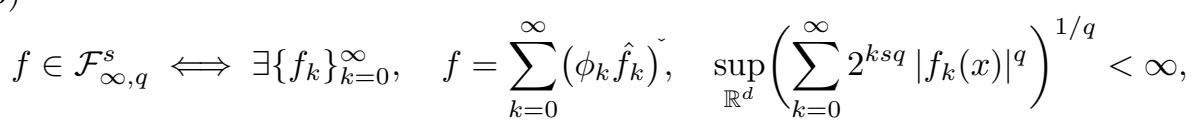


with norm

$$
\|f\|_{\mathcal{F}_{\infty, q}^{s}}=\inf \left(\sup _{\mathbb{R}^{d}}\left(\sum_{k=0}^{\infty} 2^{k s q}\left|f_{k}(x)\right|^{q}\right)^{1 / q}\right)
$$

the infimum being taken over all admissible representations.

(iv) For $1 \leq p \leq \infty$, the fractional Sobolev space $\mathcal{H}_{p}^{s}$ is defined by:

$$
f \in \mathcal{H}_{p}^{s} \Longleftrightarrow\|f\|_{\mathcal{H}_{p}^{s}}=\left(\int_{\mathbb{R}^{d}}\left|\left(\left(1+|x|^{2}\right)^{s / 2} \hat{f}(x)\right)^{p}\right|^{p} d x\right)^{1 / p}<\infty .
$$

We refer to [15, 16], 12] and 13] for background and information about the Triebel-Lizorkin, Besov, and Sobolev spaces. Because these spaces have been rediscovered (under different names) by various authors, they have a number of equivalent definitions. We collect here some of those results that will be needed in the sequel: Propositions 2.4 and 2.5 give equivalent definitions of $\mathcal{F}_{p, q}^{s}$ and $\mathcal{B}_{p, q}^{s}$, respectively, while Proposition 2.6 is a result on interpolation of Besov spaces.

The following result is proved in [15, Proposition 1, 2.3.4].

Proposition 2.4. Let $s \in \mathbb{R}, 1<p \leq \infty$, and $1<q \leq \infty$. If $f \in \mathbb{S}^{\prime}$, then

$$
\begin{gathered}
f \in \mathcal{F}_{p, q}^{s} \Longleftrightarrow \exists\left\{f_{k}\right\}_{k=0}^{\infty} \subset L^{p}, f=\sum_{k=0}^{\infty}\left(\phi_{k} \hat{f}_{k}\right), \\
\left(\int_{\mathbb{R}^{d}}\left(\sum_{k=0}^{\infty} 2^{k s q}\left|f_{k}(x)\right|^{q}\right)^{p / q} d x\right)^{1 / p}<\infty .
\end{gathered}
$$

Furthermore,

$$
\inf \left(\int_{\mathbb{R}^{d}}\left(\sum_{k=0}^{\infty} 2^{k s q}\left|f_{k}(x)\right|^{q}\right)^{p / q} d x\right)^{1 / p}
$$

is an equivalent norm on $\mathcal{F}_{p, q}^{s}$, where the infimum is taken over all admissible representations of $f$.

See [12, Theorem 2, 2.3.2] for a proof of the following result.

Proposition 2.5. Let $1 \leq p, q \leq \infty$ and $s>0$. If $f \in \mathbb{S}^{\prime}$, then

$$
\begin{gathered}
f \in \mathcal{B}_{p, q}^{s} \Longleftrightarrow \exists\left\{b_{k}\right\}_{=0}^{\infty} \subset L^{p}, f=\sum_{k=0}^{\infty} b_{k}, \\
\left(\sum_{k=0}^{\infty} 2^{k q s}\left(\int_{\mathbb{R}^{d}}\left|b_{k}(x)\right|^{p} d x\right)^{q / p}\right)^{1 / q}<\infty .
\end{gathered}
$$

Furthermore,

$$
\inf \left(\sum_{k=0}^{\infty} 2^{k q s}\left(\int_{\mathbb{R}^{d}}\left|b_{k}(x)\right|^{p} d x\right)^{q / p}\right)^{1 / q}
$$

is an equivalent norm on $\mathcal{B}_{p, q}^{s}$, where the infimum is taken over all admissible representations of $f$.

See [15, Theorem 2.4.7] for a proof of the following result.

Proposition 2.6. Let $s_{0}, s_{1} \in \mathbb{R}, 1 \leq p_{0}, q_{0}, p_{1}, q_{1} \leq \infty$ and $0<\theta<1$.

$$
\text { If } s=(1-\theta) s_{0}+\theta s_{1}, \frac{1}{p}=\frac{1-\theta}{p_{0}}+\frac{\theta}{p_{1}} \text {, and } \frac{1}{q}=\frac{1-\theta}{q_{0}}+\frac{\theta}{q_{1}} \text {, then }
$$

$$
\left(\mathcal{B}_{p_{0}, q_{0}}^{s_{0}}, \mathcal{B}_{p_{1}, q_{1}}^{s_{1}}\right)_{\theta}=\mathcal{B}_{p, q}^{s} \text {. }
$$


The next proposition collects some of the computations involved in the proofs of our results. Part (a) computes the STFT of a Gaussian with respect to a dilated Gaussian. The result is essentially the product of two Gaussians (one in time and the other in frequency). Part (b) shows that the inverse Fourier transform of the Bessel potential

$$
m_{-s}(x)=\left(1+|x|^{2}\right)^{-s / 2}
$$

is in $\mathcal{M}^{1,1}$ for $s>d$. Because $\mathcal{M}^{1,1}$ is invariant under Fourier transforms we then conclude that the Bessel potential $m_{-s}$ itself is in $\mathcal{M}^{1,1}$ for $s>d$.

Proposition 2.7. Define $g(x)=e^{-\pi x^{2}}$ and $g_{a}(x)=e^{-\frac{\pi x^{2}}{a}}$. Let

$$
G_{s}(x)=\frac{1}{(4 \pi)^{s / 2}} \frac{1}{\Gamma(s / 2)} \int_{0}^{\infty} t^{\frac{-d+s}{2}} e^{-\left(\frac{\pi x^{2}}{t}+\frac{t}{4 \pi}\right)} \frac{d t}{t}
$$

for $s, a>0$ and $x \in \mathbb{R}^{d}$. Then the following hold:

(a) $V_{g_{a}} g(x, \omega)=\left(\frac{a}{a+1}\right)^{d / 2} e^{2 \pi i \frac{x \cdot \omega}{a+1}} g_{a+1}(x) g_{\frac{a+1}{a}}(\omega)$;

(b) $V_{g} \check{m}_{-s}=(2 \pi)^{d / 2} V_{g}\left(D_{\frac{1}{2 \pi}} G_{s}\right) \in L^{1}$ for $s>d$;

(c) $m_{-s} \in \mathcal{M}^{1,1}$ for $s>d$.

Proof. (a) is an elementary, but tedious, calculation; so we will omit it.

(b) For $s>0$ we have that $\check{m}_{-s}(x)=(2 \pi)^{d / 2}\left(D_{\frac{1}{2 \pi}} G_{s}\right)(x)$ (cf. 13 Proposition 3.1.2]). Therefore:

$$
\begin{aligned}
V_{g} \check{m}_{-s}(x, \omega) & =(2 \pi)^{d / 2} V_{g}\left(D_{\frac{1}{2 \pi}} G_{s}\right)(x, \omega) \\
& =(2 \pi)^{d / 2} \int_{\mathbb{R}^{d}} D_{\frac{1}{2 \pi}} G_{s}(t) e^{-2 \pi i t \cdot \omega} \bar{g}(t-x) d t
\end{aligned}
$$

Therefore,

$$
\left\|V_{g} \check{m}_{-s}\right\|_{L^{1,1}} \leq C \int_{0}^{\infty} u^{\frac{-d+s}{2}-1}(u+2 \pi)^{d / 2} e^{-\frac{u}{4 \pi}} d u,
$$

and the last expression is finite if $s>d$.

(c) Follows from (b) and the comments above.

\section{RESUlts}

3.1. Discussion of the results. There are several embeddings between the Besov or Triebel-Lizorkin and modulation spaces that can easily be derived. These include:

(a) $\mathcal{B}_{p, q}^{s} \subset L^{p} \subset \mathcal{M}^{p, p^{\prime}}$ for $s>0,1 \leq p \leq 2$, and $1 \leq q \leq \infty$;

(b) $\mathcal{B}_{p, q}^{s} \subset L^{p} \subset \mathcal{M}^{p, p}$ for $s>0,2 \leq p \leq \infty$, and $1 \leq q \leq \infty$ (15], [16], [8]).

The embeddings we will prove are more difficult and require an appropriate norm on the Besov or Triebel-Lizorkin space in consideration, along with a correct choice of the STFT. In particular, the following equivalent forms of the STFT will be useful:

$$
\begin{aligned}
V_{g} f(x, \omega)=\left(f \cdot T_{x} \bar{g}\right) \hat{(\omega)} & =e^{-2 \pi i x \omega}\left(V_{\hat{g}} \hat{f}(\omega,-x)\right) \\
& =e^{-2 \pi i x \omega}\left(\hat{f} \cdot T_{\omega} \overline{\hat{g}}\right)=e^{-2 \pi i x \omega}\left(f *\left(M_{\omega} \bar{g}\right)(x)\right) .
\end{aligned}
$$

Our first two main results are as follows. 
Theorem 3.1. Let $1 \leq p \leq 2$ and $1 \leq q \leq \infty$. If $s>d\left(\frac{2}{p}-1\right)$, then

$$
\mathcal{B}_{p, q}^{s} \subset \mathcal{M}^{p^{\prime}, p} .
$$

Theorem 3.2. Let $1 \leq p \leq \infty$. If $s>\frac{d}{p^{\prime}}$, then

$$
\mathcal{B}_{p, p}^{s} \subset \mathcal{M}^{p, p^{\prime}} .
$$

Corollary 3.3. If $2 \leq p \leq \infty$ and $s>d\left(1-\frac{2}{p}\right)$, then

$$
\mathcal{B}_{p, p}^{s} \subset \mathcal{M}^{p, p^{\prime}} .
$$

Theorem 3.2, which recovers and extends the embedding in [10], follows by identifying $\mathcal{F}_{p, p}^{s}$ with $\mathcal{B}_{p, p}^{s}$ and by using Proposition 2.4 as the appropriate definition of $\mathcal{B}_{p, p}^{s}$. However, it does not include the fact that $\mathcal{B}_{2,2}^{s} \subset L^{2}=\mathcal{M}^{2,2}$ for $s \geq 0$. This last embedding is obtained in Corollary 3.3 by using complex interpolation methods.

Theorem 3.4. If $1 \leq p, q, r \leq \infty$ with $\frac{1}{p}+\frac{1}{q}=1+\frac{1}{r}$, then

$$
\mathcal{H}_{p}^{s} \subset \mathcal{M}^{r, 1} \quad \text { for } \quad s>d .
$$

Theorem 3.4 yields an embedding of the fractional Sobolev space (or Bessel potential space) into the modulation space. This can also be seen as an embedding of the Triebel-Lizorkin space $\mathcal{F}_{p, 2}^{s}$ into the modulation space since $\mathcal{F}_{p, 2}^{s}=\mathcal{H}_{p}^{s}$ for $1<p<\infty$ (15], [16] and [12]).

The next corollary holds only in dimension one and gives a useful sufficient condition on a function to be in $\mathcal{M}^{1,1}$. The corollary follows from the identification of the Bessel potential space $\mathcal{H}_{p}^{2}$ with the Sobolev space $\mathcal{W}^{2, p}$ obtained by imposing that $f$ and its first two derivatives belong to $L^{p}$, for $p=1$ or $p=\infty$.

Corollary 3.5. If $d=1$, then

$$
\mathcal{W}^{2,1}=\left\{f \in L^{1}: f^{\prime}, f^{\prime \prime} \in L^{1},\|f\|=\sum_{k=0}^{2} \int_{\mathbb{R}}\left|f^{(k)}(x)\right| d x<\infty\right\} \subset \mathcal{M}^{1,1}
$$

and

$$
\mathcal{W}^{2, \infty}=\left\{f \in L^{\infty}: f^{\prime}, f^{\prime \prime} \in L^{\infty},\|f\|=\sum_{k=0}^{2} \sup _{\mathbb{R}}\left|f^{(k)}(x)\right|<\infty\right\} \subset \mathcal{M}^{\infty, 1} .
$$

Note added. After completing this work, we learned that the embedding (16) had previously been conjectured by H. Feichtinger and a proof different from ours was obtained by K. Gröchenig.

\section{Proofs}

4.1. Proof of Theorem 3.1. Let $f \in \mathcal{B}_{p, q}^{s}$, and use (8) to write $f=\sum b_{k}$ where $b_{k} \in L^{p}, \operatorname{supp}\left(\hat{b}_{k}\right) \subset\left\{|x| \leq 2^{k}\right\}$, and $\|f\|_{\mathcal{B}_{p, q}^{s}} \sim \inf \left(\sum_{k=0}^{\infty} 2^{k s q}\left\|b_{k}\right\|_{L^{p}}^{q}\right)^{1 / q}$, where the infimum is over all possible such representations of $f$. Given $g \in \mathbb{S}$, we have using (11):

$$
V_{g} f(x, \omega)=\sum_{k=0}^{\infty} e^{-2 \pi i x \omega}\left(\hat{b}_{k} \cdot T_{\omega} \overline{\hat{g}}\right)(x) .
$$


Hence by the Hausdorff-Young inequality,

$$
\left\|V_{g} f(\cdot, \omega)\right\|_{L^{p^{\prime}}} \leq \sum_{k=0}^{\infty}\left\|\left(\hat{b}_{k} \cdot T_{\omega} \overline{\hat{g}}\right)\right\|_{L^{p^{\prime}}} \leq \sum_{k=0}^{\infty}\left\|\hat{b}_{k} \cdot T_{\omega} \overline{\hat{g}}\right\|_{L^{p}}
$$

Therefore, by Minkowski's inequality,

$$
\left\|V_{g} f\right\|_{L^{p^{\prime}, p}} \leq \sum_{k=0}^{\infty}\left(\int_{\mathbb{R}^{d}}\left\|\hat{b}_{k} \cdot T_{\omega} \overline{\hat{g}}\right\|_{L^{p}}^{p} d \omega\right)^{1 / p}=\|\hat{g}\|_{L^{p}} \sum_{k=0}^{\infty}\left\|\hat{b}_{k}\right\|_{L^{p}} .
$$

Now, $\hat{b}_{k}$ has compact support $K \subset\left\{|x| \leq 2^{k}\right\}$. Since $1 \leq p \leq 2 \leq p^{\prime}$, we have $L^{p^{\prime}}(K) \subset L^{p}(K)$, and

$$
\left\|\hat{b}_{k}\right\|_{L^{p}} \leq C 2^{k d\left(\frac{1}{p}-\frac{1}{p^{\prime}}\right)}\left\|b_{k}\right\|_{L^{p}}
$$

where $C$ is the volume of the ball of center 0 and radius 1 . Substituting (19) into (18) and applying Hölder's inequality yields

$$
\begin{aligned}
\left\|V_{g} f\right\|_{L^{p^{\prime}, p}} & \leq C\left(\sum_{k=0}^{\infty} 2^{k s q}\left\|b_{k}\right\|_{L^{p}}^{q}\right)^{1 / q}\left(\sum_{k=0}^{\infty} 2^{k q^{\prime} d\left(\frac{1}{p}-\frac{1}{p^{\prime}}-\frac{s}{d}\right)}\right)^{1 / q^{\prime}} \\
& \leq C\|f\|_{\mathcal{B}_{p, q}^{s}}\left(\sum_{k=0}^{\infty} 2^{k q^{\prime} d\left(\frac{1}{p}-\frac{1}{p^{\prime}}-\frac{s}{d}\right)}\right)^{1 / q^{\prime}}
\end{aligned}
$$

The last term in (20) is finite if and only if $s>d\left(\frac{1}{p}-\frac{1}{p^{\prime}}\right)$.

4.2. Proof of Theorem 3.2. The case $p=1$ is trivial; so assume $1<p \leq \infty$. By (77) with $p=q$ we obtain an equivalent norm for $\mathcal{F}_{p, p}^{s}=\mathcal{B}_{p, p}^{s}$. Let $f \in \mathcal{B}_{p, p}^{s}$. Then $f=\sum_{k=0}^{\infty}\left(\phi_{k} \hat{f}_{k}\right)$. Let $g(x)=e^{-\pi x^{2}}$. Then, using (11),

$$
V_{g} f(x, \omega)=\sum_{k=0}^{\infty} f_{k} *\left(\phi_{k} \cdot T_{\omega} \overline{\hat{g}}\right)(x)
$$

So by Young's convolution inequality,

$$
\left\|V_{g} f(., \omega)\right\|_{L^{p}} \leq \sum_{k=0}^{\infty}\left\|f_{k}\right\|_{L^{p}}\left\|\left(\phi_{k} \cdot T_{\omega} \overline{\hat{g}}\right)\right\|_{L^{1}} .
$$

Hence, by Minkowski's inequality and Hölder's inequality,

$$
\begin{aligned}
\left\|V_{g} f\right\|_{L^{p, p^{\prime}}} & \leq \sum_{k=0}^{\infty}\left\|f_{k}\right\|_{L^{p}}\left(\int_{\mathbb{R}^{d}}\left(\left\|\left(\phi_{k} \cdot T_{\omega} \overline{\hat{g}}\right)\right\|_{L^{1}}\right)^{p^{\prime}} d \omega\right)^{1 / p^{\prime}} \\
& \leq\left(\sum_{k=0}^{\infty} 2^{k s p}\left\|f_{k}\right\|_{L^{p}}^{p}\right)^{1 / p}\left(\sum_{k=0}^{\infty} 2^{-k s p^{\prime}} \int_{\mathbb{R}^{d}}\left\|\left(\phi_{k} \cdot T_{\omega} \overline{\hat{g}}\right)\right\|_{L^{1}}^{p^{\prime}} d \omega\right)^{1 / p^{\prime}}
\end{aligned}
$$

and therefore

$$
\|f\|_{\mathcal{M}^{p, p^{\prime}}} \leq\|f\|_{\mathcal{B}_{p, p}^{s}}\left(\sum_{k=0}^{\infty} 2^{-k s p^{\prime}} \int_{\mathbb{R}^{d}} \|\left(\phi_{k} \cdot T_{\omega} \overline{\hat{g}}\right)_{\|_{L^{1}}^{p^{\prime}}}^{p^{\prime}} d \omega\right)^{1 / p^{\prime}} .
$$


Now we will estimate the terms on the right-hand side of (21). Setting $g_{k}(x)=$ $g\left(2^{-k+1} x\right)$, we have:

$$
\begin{aligned}
\left(\int_{\mathbb{R}^{d}}\left\|\left(\phi_{k} \cdot T_{\omega} \overline{\hat{g}}\right)\right\|_{L^{1}}^{p^{\prime}} d \omega\right)^{1 / p^{\prime}} & =\left\|V_{g} \check{\phi}_{k}\right\|_{L^{1, p^{\prime}}} \\
& =\left\|V_{g_{k}} \check{\phi}_{1}\left(2^{k-1} \cdot, 2^{-k+1} \cdot\right)\right\|_{L^{1, p^{\prime}}} \\
& =2^{d(-k+1)} 2^{d(k-1) / p^{\prime}}\left\|V_{g_{k}} \check{\phi}_{1}\right\|_{L^{1, p^{\prime}}} \\
& \leq C_{1} 2^{d / p} 2^{-k d / p}\left\|V_{g_{k}} g\right\|_{L^{1,1}}\left\|V_{g} \check{\phi}_{1}\right\|_{L^{1, p^{\prime}}},
\end{aligned}
$$

the last inequality following from the independence of the definition of the modulation space with respect to the window used to compute the STFT; cf. 8 8 , Proposition 11.3.2]. Using Proposition 2.7 with $a=2^{k-1}$, we have that

$$
\left\|V_{g_{k}} g\right\|_{L^{1,1}}=\left\|V_{g_{a}} g\right\|_{L^{1,1}}=\left(1+2^{2 k-2}\right)^{d / 2} .
$$

Combining (21), (221) and (231) yields:

$$
\|f\|_{\mathcal{M}^{p, p^{\prime}}} \leq C_{2}\|f\|_{\mathcal{B}_{p, p}^{s}}\left(\sum_{k=0}^{\infty} 2^{-k p^{\prime}(s-d+d / p)}\right)^{1 / p^{\prime}} .
$$

The last term in the right-hand side of (24) is finite if and only if $s>d\left(1-\frac{1}{p}\right)=\frac{d}{p^{\prime}}$.

4.3. Proof of Corollary 3.3. We will prove this part by interpolating between the cases $p=2, s_{0} \geq 0$ and $p=\infty, s_{1}>d$. In particular, we trivially have

$$
\mathcal{B}_{2,2}^{s_{0}}=\mathcal{F}_{2,2}^{s_{0}}=\mathcal{H}_{2}^{s_{0}} \subset L^{2}=\mathcal{M}^{2,2} \quad \text { for } \quad s_{0} \geq 0,
$$

and applying Theorem 3.2 to $p=\infty$ yields:

$$
\mathcal{B}_{\infty, \infty}^{s_{1}} \subset \mathcal{M}^{\infty, 1} \quad \text { for } \quad s_{1}>d \text {. }
$$

Hence, to complete the proof we apply (2) and (9) with $s_{0} \geq 0, s_{1}>d, p_{0}=q_{0}=2$, and $p_{1}=q_{1}=\infty$.

4.4. Proof of Theorem 3.4. Let $m_{-s}(x)=\left(1+|x|^{2}\right)^{-s / 2}$ and $g(x)=e^{-\pi x^{2}}$. Then for $f \in \mathcal{H}_{p}^{s}$ we have:

$$
V_{g} f(x, \omega)=e^{-2 \pi i x \omega}\left(\hat{f} \cdot T_{\omega} \overline{\hat{g}}\right)(x)=e^{-2 \pi i x \omega}\left(\left(\hat{f} m_{s}\right)^{\check{L}} *\left(m_{-s} \cdot T_{\omega} \overline{\hat{g}}\right) \check{(x)} .\right.
$$

Hence, by Young's convolution inequality,

$$
\left\|V_{g} f(\cdot, \omega)\right\|_{L^{r}} \leq\left\|\left(\hat{f} m_{s}\right)^{\check{1}}\right\|_{L^{p}}\left\|\left(m_{-s} \cdot T_{\omega} \overline{\hat{g}}\right)\right\|_{L^{q}},
$$

and so

$$
\left\|V_{g} f\right\|_{L^{r, 1}} \leq\|f\|_{\mathcal{H}_{p}^{s}}\left(\int_{\mathbb{R}^{d}}\left(\int_{\mathbb{R}^{d}}\left|\left(m_{-s} \cdot T_{\omega} \overline{\hat{g}}\right)(x)\right|^{q} d x\right)^{1 / q} d \omega\right)=\|f\|_{\mathcal{H}_{p}^{s}}\left\|V_{g} \check{m}_{-s}\right\|_{L^{q, 1}}
$$

Using (10), we have that $\left\|V_{g} \check{m}_{-s}\right\|_{L^{q, 1}}<\infty$ for $s>d$, which concludes the proof.

\subsection{Proof of Corollary 3.5.}

Proof. If $d=1$, then $\mathcal{H}_{1}^{2}=\mathcal{W}^{2,1}$ and $\mathcal{H}_{\infty}^{2}=\mathcal{W}^{2, \infty}$ [13], and so the proof follows from Theorem 3.4 with $d=1$. 


\section{ACKNOWLEDGMENT}

The author would like to thank Prof. Christopher Heil for invaluable discussions and his support. He would also like to thank Profs. Hans G. Feichtinger, Karlheinz Gröchenig and Joachim Toft for fruitful discussions.

\section{REFERENCES}

[1] H. G. Feichtinger, Modulation spaces on locally Abelian groups, Technical Report, University of Vienna, 1983.

[2] H. G. Feichtinger and K. Gröchenig, Banach spaces related to integrable group representations and their atomic decompositions. I, J. Funct. Anal. 86:307-340, 1989. MR 91g:43011

[3] H. G. Feichtinger and K. Gröchenig, Banach spaces related to integrable group representations and their atomic decompositions. II, Monatsh. Math. 108:129-148, 1989. MR 91g:43012

[4] G. B. Folland, Harmonic Analysis in Phase Space, Annals of Mathematics Studies, vol. 122, Princeton University Press, Princeton, NJ, 1989. MR 92k:22017

[5] Y. V. Galperin and K. Gröchenig, Uncertainty principles as embeddings of modulation spaces, J. Math. Anal. Appl. 274(1):181-202, 2002. MR 2003h:46040

[6] P. Gröbner, Banachräume Glatter Funktionen und Zerlegungsmethoden, Ph.D. Thesis, University of Vienna, 1983.

[7] K. Gröchenig, An uncertainty principle related to the Poisson summation formula, Studia Math. 121:87-104, 1996. MR 98a:42007

[8] K. Gröchenig, Foundations of Time-Frequency Analysis, Applied and Numerical Harmonic Analysis, Birkhäuser, Boston, MA, 2001. MR 2002h:42001

[9] K. Gröchenig and C. Heil, Modulation spaces and pseudodifferential operators, Integral Equations Operator Theory 34:439-457, 1999. MR 2001a:47051

[10] C. Heil, J. Ramanathan, and P. Topiwala, Singular values of compact pseudodifferential operators, J. Funct. Anal. 150:426-452, 1997. MR 98k:47102

[11] J. A. Hogan and J. D. Lakey, Embeddings and uncertainty principles for generalized modulation spaces, in "Sampling Theory and Its Applications," J. J. Benedetto and P. Ferreira, eds., Modern Sampling Theory - Mathematics and Applications, Birkhäuser, Boston, MA, 2001. MR 2003a:94003

[12] T. Runst and W. Sickel, Sobolev Spaces of Fractional Order, Nemytskij Operators, and Nonlinear Partial Differential Equations, Walter de Gruyter, Berlin, 1996. MR 98a:47071

[13] E. M. Stein, Singular Integrals and Differentiability Properties of Functions, Princeton University Press, Princeton, NJ, 1970. MR 44:7280

[14] J. Toft, Embeddings for modulation spaces and Besov spaces, Research Report 2001:11, Blekinge Institute of Technology, 2002.

[15] H. Triebel, Theory of Function Spaces, Monographs in Mathematics, No. 78, Birkhäuser Verlag, Basel, 1983. MR 86j:46026

[16] H. Triebel, Theory of Function Spaces II, Monographs in Mathematics, No. 84, Birkhäuser Verlag, Basel, 1992. MR 93f:46029

School of Mathematics, Georgia Institute of Technology, Atlanta, Georgia 30332 0160

E-mail address: okoudjou@math.gatech.edu

Current address: Department of Mathematics, Malott Hall, Cornell University, Ithaca, New York, 14853-4201

E-mail address: kasso@math.cornell.edu 